\section{Allergic Gingivostomatitis (Due to Gum Chewing)}

by

Donald A. KeRR, * D.D.S., M.S.

Kenneth D. MCClatchey,** D.D.S., M.S.

JoSEPH A. REGEZI, ${ }^{* *}$ D.D.S., M.S.

IN 1968 A NUMBER OF CASES of a characteristic, but unusual, type of gingivostomatitis appeared throughout the country. Most periodontists had several cases on referral from general practitioners. Because of the classical appearance of this gingivostomatitis, it was readily recognized that this was a specific disease process and that there must be a common etiology. In a few months the authors accumulated 12 patients ranging in age from 11 to 58 years. Eight were females and four were males. This group of patients stimulated an investigation of the problems of etiology and treatment.

\section{Clinical Signs and Symptoms}

The patients presented with an intense hyperemia of the free and attached gingiva. The gingiva was slightly to moderately edematous, and this often intensified the stippling. In severe cases the buccal and vestibular mucosa was edematous and hyperemic. In very severe cases the vestibular mucosa was eroded. The tongue was involved in all cases by the loss of the filiform papillae, producing a very red, clean surface. Due to depapillation the fungiform papillae were exposed, enlarged and hyperemic. In patients with furrowed tongue, the folds were enlarged and the furrows deepened. Due to the swelling, the tongue usually developed lateral indentations. The lips were affected in all patients by an atrophic, dry, scaly cheilitis with some intensification of color. In advanced cases there was cracking of the lips. The most severe involvement was the angular cheilitis, with fissuring and maceration of the mucosa at the commissure. This appeared as a typical perleche-type of lesion. The signs varied in severity, but all patients presented the full triad of involvement. All complained of sore burning tongue and gingiva. Most complained of sensitivity to dentifrices and highly seasoned foods. Many had discontinued the use of dentifrices because of the discomfort they produced.

*Professor and Chairman, Department of Oral Pathology, School of Dentistry, University of Michigan, Ann Arbor, Michigan.

**Department of Oral Pathology, School of Dentistry, University of Michigan, Ann Arbor, Michigan.

\section{Past History}

Most of our patients reported a sudden onset of the disease. They said it had been present from three months to two years with exacerbations and remissions, but never complete remission. The patients sometimes associated remissions with some form of therapy.

Various treatments had been used. Patients had been treated with vitamin B because the lip lesions were similar to those due to riboflavin deficiency. Others had been treated with vitamin $B_{12}$ and iron compounds because the tongue changes resembled some of those found in anemia. Mycostatin also was used because the perleche-type lesions resembled the angular cheilitis seen in Candida infections in this location. ${ }^{1}$ Gingivectomy also had been done on some patients. ${ }^{2}$

\section{Clinical Laboratory Findings}

Blood studies, consisting of hemoglobin determination, hematocrit, red blood cell and white cell count, including differential WBC, were done on all patients. All results were within normal limits, except for a tendency for slight eosinophilia in some patients. Blood chemistry studies on the first two patients were within normal limits and thus were not completed on the others. Bacterial studies included smears, aerobic and anaerobic blood agar cultures, cultures for fungi, and in two cases viral cultures. The smears and blood agar cultures showed a variety of organisms which constituted normal oral flora. The cultures for monilia were positive initially in two cases, with a third becoming positive on subsequent culture.

\section{HistopathOLOGY}

All biopsies of the gingiva demonstrated the same changes but of varying severity. The epithelium was

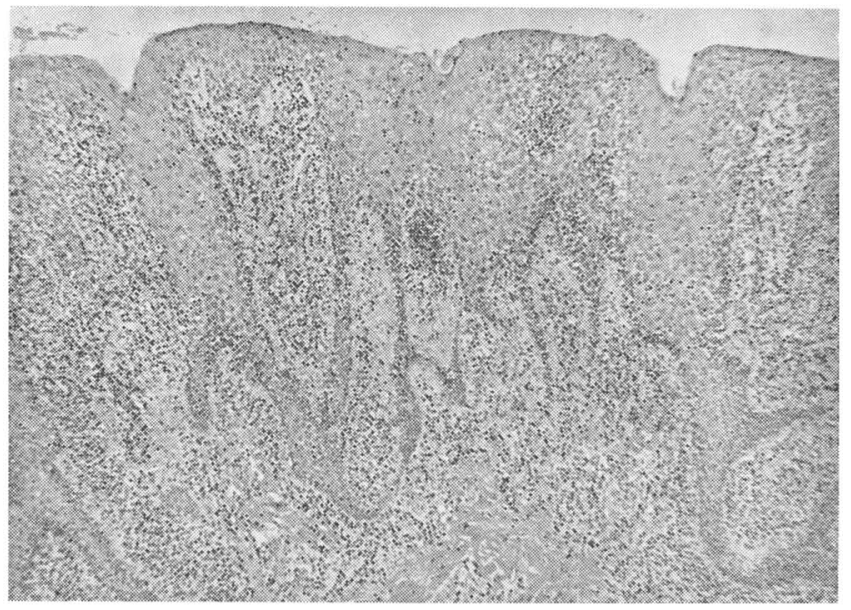

FIGURE 1. Hyperplasia of epithelium with preservation of stipples. Individual cell keratinization of squamous cell at base of stipples. Close proximity of connective tissue papillae to the surface. Heavy plasma cell infiltration in the submucosa with inflammatory infiltration in the epithelium. $H \& E . X 52$. 

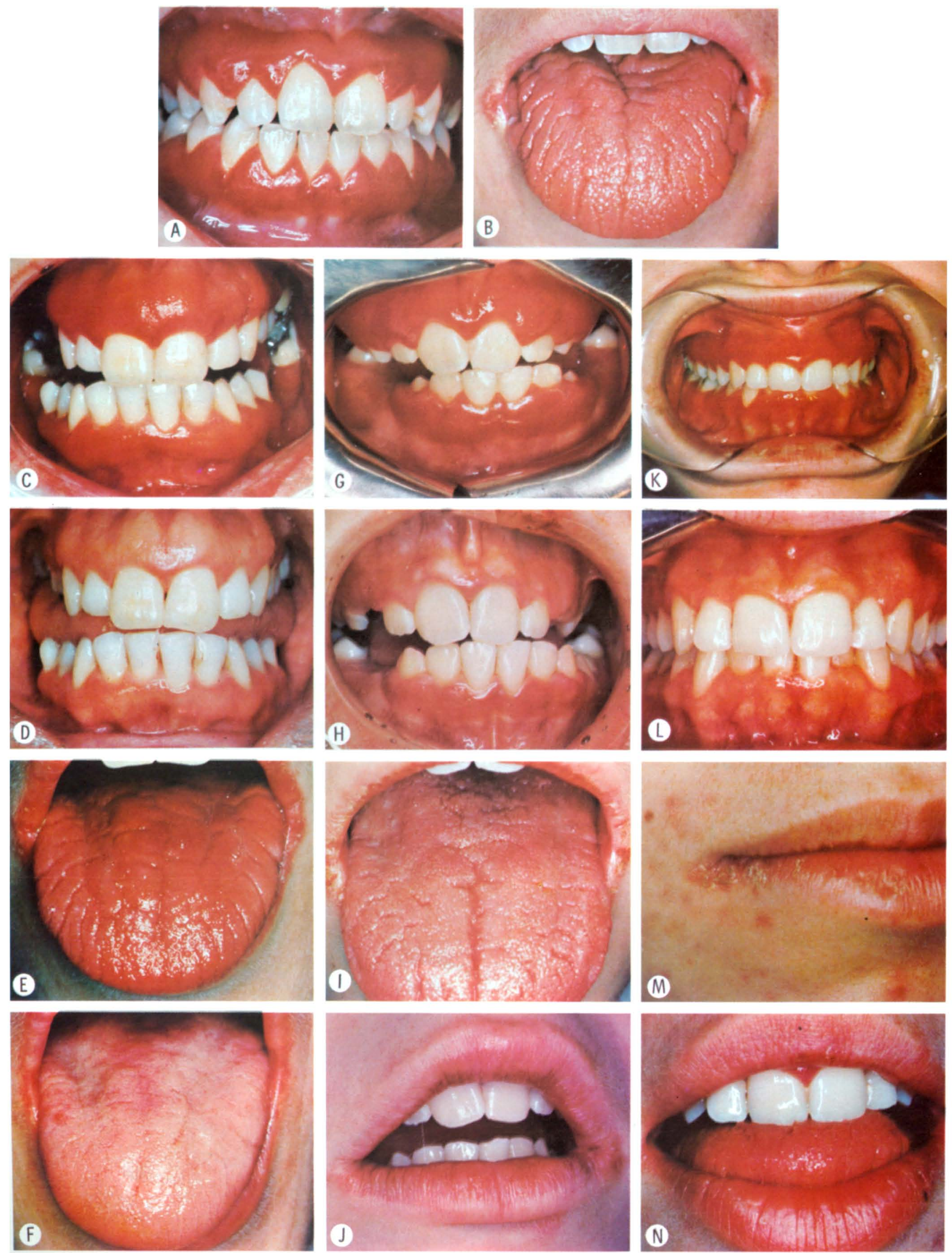
Case 1. (30-year-old female).

FIgURE A. Typical gingival picture shows intense red color of free and attached gingiva. Note the sharp line of demarcation of gingival mucosa from alveolar mucosa. Swelling of marginal and papillary gingiva.

FIGURE B. This demonstrates the typical angular cheilitis. The tongue shows loss of filiform papillae, swelling of fungiform papillae, exaggeration of fissures due to swelling, and marginal hyperplasia.

Case II. (28-year-old female).

FIGURE C. Typical gingival change. Note persistence of stippling of swollen, hyperemic gingiva.

FIGURE D. Gingiva after patient had stopped chewing gum for four weeks.

FIGURE E. Typical lip and tongue changes.

Figure F. Lips and tongue four weeks after patient stopped chewing gum. Angular cheilitis has disappeared. Marked reduction in fissuring due to decrease in swelling. Fungiform papillae are reduced to normal and filiform papillae are regenerating.

Case III. (11-year-old female).

FIGURE G. Typical gingival hyperemia and swelling of free and attached gingiva. Some persistence of stippling.

Figure H. Nearly normal gingival tissue four weeks after cessation of gum chewing.

FIGURE I. Typical cheilitis showing maceration at commissure with atrophy and crusting of the vermilion. Tongue shows swelling with exaggeration of fissures, depapillation, and enlarged fungiform papillae.

FIGURE J. Normal lip four weeks after patient stopped chewing gum.

Case IV. (22-year-old white female).

FIGURE K. Marked hyperemia of free and attached gingiva. Moderate swelling of the free and papillary gingiva.

FIGURE L. Normal gingiva four weeks after the patient stopped chewing gum.

FIGURE M. Crusting of mucosa and skin at commissure. Dry scaly lesions extend over vermilion almost to midline.

FIGURE N. Lips have returned to normal in two weeks even though tongue and gingiva show some change still present.

mildly hyperplastic with areas of individual cell keratinization about which there were areas of liquefaction forming microvesicles. The microvesicles contained leukocytes. Leukocytic infiltration throughout the thickness of the epithelium was permitted by the marked spongiosis and alterations in the basal cell layer. There was a heavy plasma cell infiltration of the submucosa of such intensity that most of the structures in the area were obliterated. Deeper in the tissue focal accumulations were seen about dilated vascular spaces. Some dilated vessels in the connective tissue papillae nearly reached the surface due to the thinness of the overlying epithelium. The heavy infiltration of plasma cells in the gingiva was the reason for designating the condition as plasma cell gingivitis. ${ }^{1}$ The plasma cells also suggested that the condition might be allergic in nature (Fig. 1).

The sudden occurrence in the population, the distribution of the lesions, and the history of sensitivity to dentifrices, all suggested an allergic response to some substance taken into the mouth. Because of this speculation, a dietary history was taken on each patient, and for one week everything taken into the mouth was recorded. This included food, drinks, dentifrices, mouth washes, tobacco, medications, gum and candy. One patient already had been on an elimination diet before presenting for consultation.

\section{TREATMENT}

On presentation the patients had been treated with a large variety of therapeutic agents, which included vitamins $\mathrm{B}$ and $\mathrm{C}$, iron, antibiotics including Mycostatin, Kenalog and Orabase, hydrogen peroxide, and various mouth washes. For some of our first patients we prescribed syrup of Phenergan, elixir of Benadryl, steroid creams in Sta-guard carriers, and Mycostatin if the patient had a positive culture for Candida. Some patients who were taking birth control pills discontinued their use. However, due to the fact that our patients included an 11-year-old girl, a 58-year-old woman, and four males, it was felt that the pill was not a factor. The regimen on which patients were placed produced some reduction in severity of the condition in most patients, but it did not provide a cure.

In reviewing the dietary histories, we observed that the one thing common to all patients was habitual gum chewing. We therefore took all of them off chewing gum, and an immediate response became evident. Within four weeks all patients had complete cure of the lip and tongue changes, and the gingiva had returned to the previous state of health. When patients had a marked reduction of symptoms, some were given gum to chew for 15 minutes. This caused almost immediate burning of the lips and tongue and redness of the gums.

After the patients had returned to normal, they were dismissed and told to return if they had any recurrence of symptoms. Only one returned, and he presented with an exacerbation. He denied chewing gum but did admit to consumption of a large number of candy suckers over the previous weekend (Plate I).

\section{DISCUSSION}

It was apparent from our results that there was some agent in chewing gum, and perhaps some forms of candy, which served as an allergin to produce a contact gingivostomatitis.

Almost as suddenly as initial cases appeared, new cases did not present. We have the feeling that the man- 
ufacturers used some new ingredient for a short time and discontinued its use to return to their original formula; therefore, there was a reduction in new cases. Because gum is a very complex formula of chicle, sweeteners, and flavoring agents, there are many possibilities as to what the antigen might be. Because we know of a few cases in which the patients did not give a history of gum chewing, and because of the exacerbation produced in one of our patients by "suckers," we anticipate that the allergin may be used in some types of hard candies. This should be considered if the patients do not chew gum.

\section{SUMMARY}

In the past few years clinicians have seen a large number of patients with a form of gingivostomatitis which had previously not presented. The clinical and histologic features were constant and classical for this condition. This paper presents the clinical features of the disease process and demonstrates that it is a hypersensitivity reaction to some constituent of chewing gum and/or hard candy.

\section{BIBLIOGRAPHY}

1. Vickers, R. A. and Hudson, Carolyn D.: A Clinicopathologic Investigation of "Plasma Cell Gingivitis." IADR Abstr. 755, March 1971.

2. Owings, J. R.: An Atypical Gingivostomatitis: A Report of Four Cases. J. Periodont., 40:538, 1969.

3. Figley, K. D.: Karaya Gum (Indian gum) Hypersensitivity. J. Amer. Med. Assn., 114:9, 1947.

4. Miller, Jerome: Cheilitis from Sensitivity to Oil of Cinnamon Present in Bubble Gum. J. Amer. Med. Assn., 116:131, 1941.

5. Poswillo, D.: Plasmacytosis of the Gingiva. Brit. J. O. Surg., 5:194, 1968.

6. Sugarman, M. M.: Contact Allergy Due to Mint Chewing Gum. Oral Surg., 3:1145, 1950.

7. Yeretsky, W.: Contact Allergy Due to Chewing Gum. A Case Report. Mich. Dent. A. J., 22:275, 1940.

\section{Abstracts}

\section{Comparative Study of the Influence of Pregnancy and Oral Contraceptives on the Gingivae}

El-Ashiry, G., El-Kafrawy, A. H., Nasr, M. F., and Younis, N. Oral Surg. 30:472, October, 1970

A total of 120 pregnant and 125 other females taking oral contraceptives was used, along with a control group of 50 women neither pregnant nor taking any hormones. Both a gingival index and a calculus index were made of the twelve anterior teeth. The pregnant women were divided into groups according to the trimester of pregnancy, while the women on oral contraceptives were divided according to length of time they had been taking the pill. Averages were made of the calculus scores and gingival scores and the "t" test was applied for analysis. It was found that gingival indices of women taking "the pill" for three months were similar to the condition of women in the first trimester of pregnancy while those taking the pill for six to nine months had gingival indices similar to those in the third trimester of pregnancy. The women taking oral contraceptives for one or two years showed a gingival index similar to the first or second trimester of pregnancy. It was suggested that these changes may be due to degranulation of gingival mast cells although this supposition needs to be studied. Cairo University, School of Dentistry, Cairo, Egypt, U.A.R.

\section{Nature of Contacts in Centric Occlusion in 32 Adults}

Anderson, J. R., Jr. and Hyers, G. E.

J. Dent. Res. 50:7, January-February, 1971

The 32 patients had complete dentitions with the exception of some missing third molars and a few areas which could be restored with fixed prosthesis. Their oral hygiene was good, no periodontal pathology was present, and there were no abnormal wear facets. Only 8 out of 32 patients had contact of all teeth in centric occlusion (maximum intercuspation position). The anterior teeth contacts were on inclined planes with the labial margin of the incisal edge of the mandibular tooth in contact with the lingual surface of the maxillary tooth. Out of 565 posterior tooth contacts there were 37 flat plane (bottom of fossa, marginal ridge or cusp tip), 16 on one inclined plane, 153 on two inclined planes and 335 with combinations of these contacts. Ideal occlusion was not seen in any of the dentitions examined. Crown and Bridge Department, University of Michigan School of Dentistry, Ann Arbor, Michigan 48104.

Identification and PURIfication of Hyaluronidases from Cultured Dental Plaque Material

Nord, C-E., Söder, P-O, Lindquist, L. and Frostell, G.

Odont. Revy 21:13, \#1, 1970

Dental plaque collected from ten healthy patients was placed in suspension and then cultured in a special medium. After using the Hultin method to determine enzyme activity, a straight line relationship was seeen in plotting the viscosity reciprocal versus time and the hyaluronidase activity versus the supernatant concentration. The greatest hyaluronidase activity was seen in the range of $\mathrm{pH} 5.0$ to a peak of 6.6 with no activity evident at 4.0. Heating the supernatant at $37^{\circ} \mathrm{C}$ for 30 minutes did not affect the activity but it decreased to 9 percent at $50^{\circ} \mathrm{C}$ and showed no activity at $60^{\circ} \mathrm{C}$. The hyaluronidase from cultured plaque material were then put through a purification procedure and two distinct hyaluronidase fractions were isolated. Hyaluronidase activity in the cultured plaque material was notably greater than in dental plaque material and both fractions were obtained as rather pure and potent enzymes. Department of Oral Microbiology, Karolinska Institutet, Tandläkarhögskolan, Box 3207, S-103 64, Stockholm, Sweden. 